Supporting Information

Bridging the Gap between Macroscale Drug Delivery Systems and Nanomedicines: A Nanoparticle-assembled Thermosensitive Hydrogel for Peritumoral Chemotherapy

Pingsheng Huang ${ }^{1 a}$, Huijuan Song ${ }^{1 a}$, Yumin Zhang ${ }^{2}$, Jinjian Liu ${ }^{2}$, Ju Zhang ${ }^{1}$, Weiwei Wang ${ }^{{ }^{*}}$, Jianfeng Liu ${ }^{2}$, Chen Li $^{1}$, Deling Kong ${ }^{1 *}$

1 Tianjin Key Laboratory of Biomaterial Research, Institute of Biomedical Engineering, Chinese Academy of Medical Sciences and Peking Union Medical College, Tianjin 300192, China

${ }^{2}$ Tianjin Key Laboratory of Radiation Medicine and Molecular Nuclear Medicine, Institute of Radiation Medicine, Chinese Academy of Medical Sciences and Peking Union Medical College, Tianjin 300192, China

*Corresponding Author

E-mail: wwwangtj@163.com; kongdeling@nankai.edu.cn. 

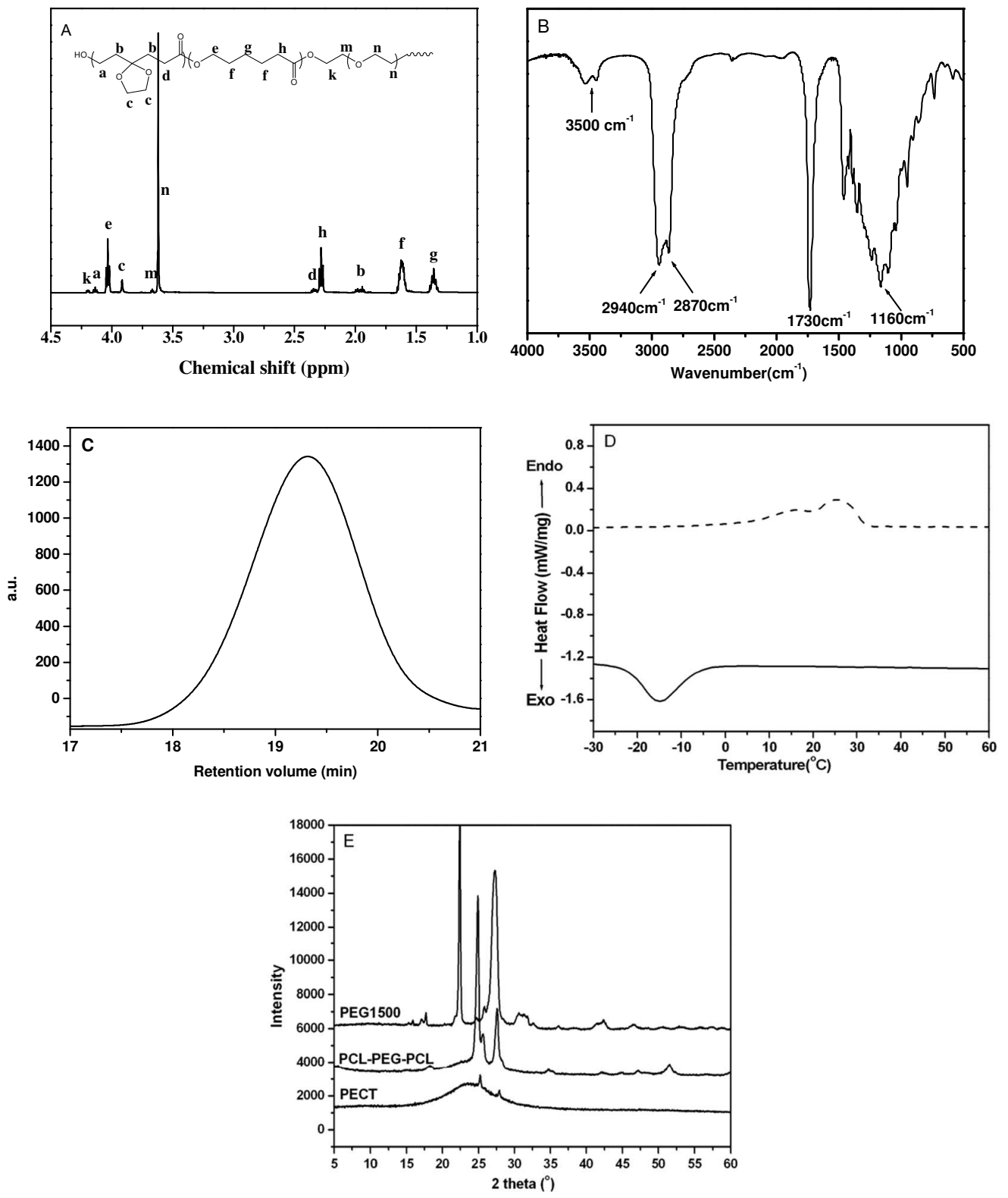

Fig. S1 The ${ }^{1} \mathrm{H}$ NMR spectrum (A), FI-IR spectrum (B), GPC curve (C), DSC profile (D) and XRD spectrum (E) of PECT copolymer. 


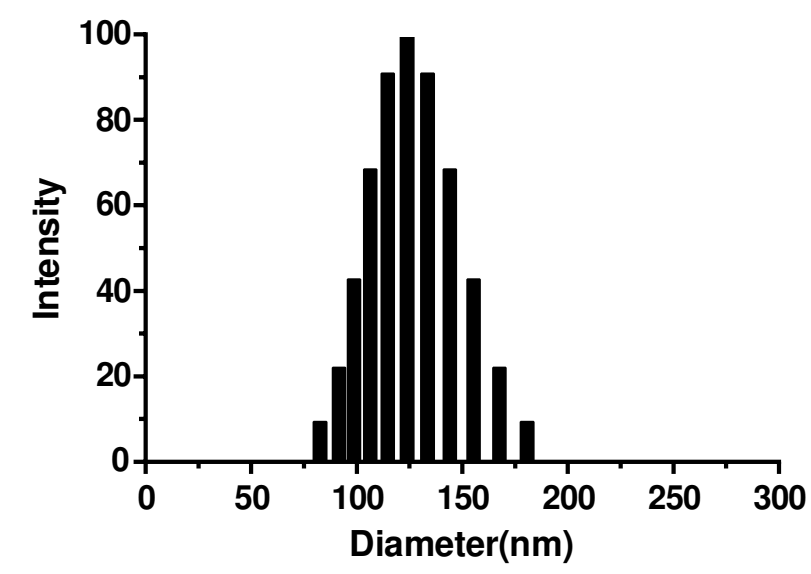

Fig. S2 The particle size of fresh prepared PECT/Dox NPs at mass concentration of $1 \%$.

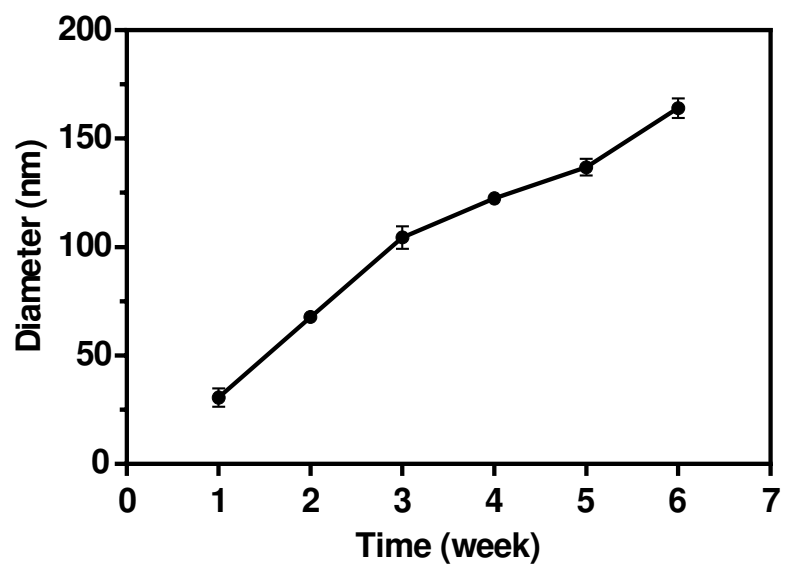

Fig. S3 Variation of particle size in the release medium in vitro without changing release medium

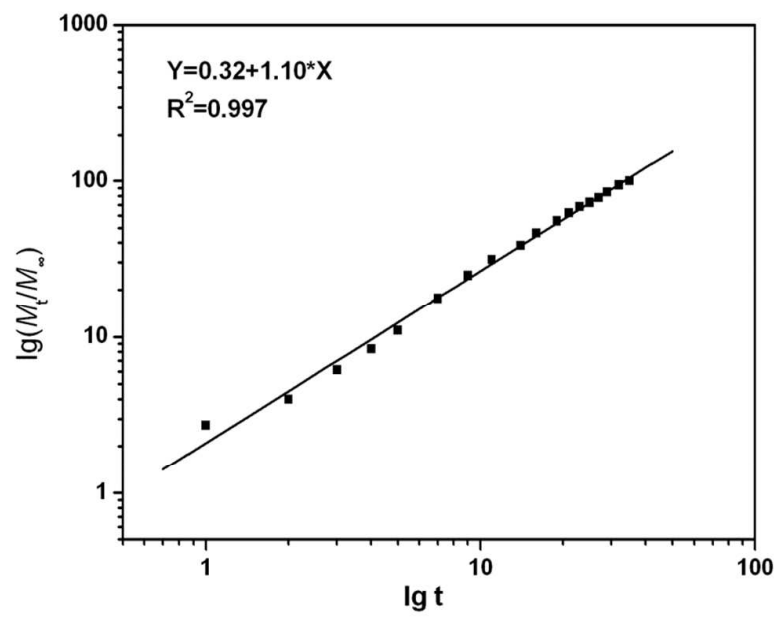

Fig. S4 The linear fitting curve of $\lg \left(M_{\mathrm{t}} / M_{\infty}\right)$ as a function of $\lg t$. 


\section{Synthesis and characterization of PECT-RB conjugates}

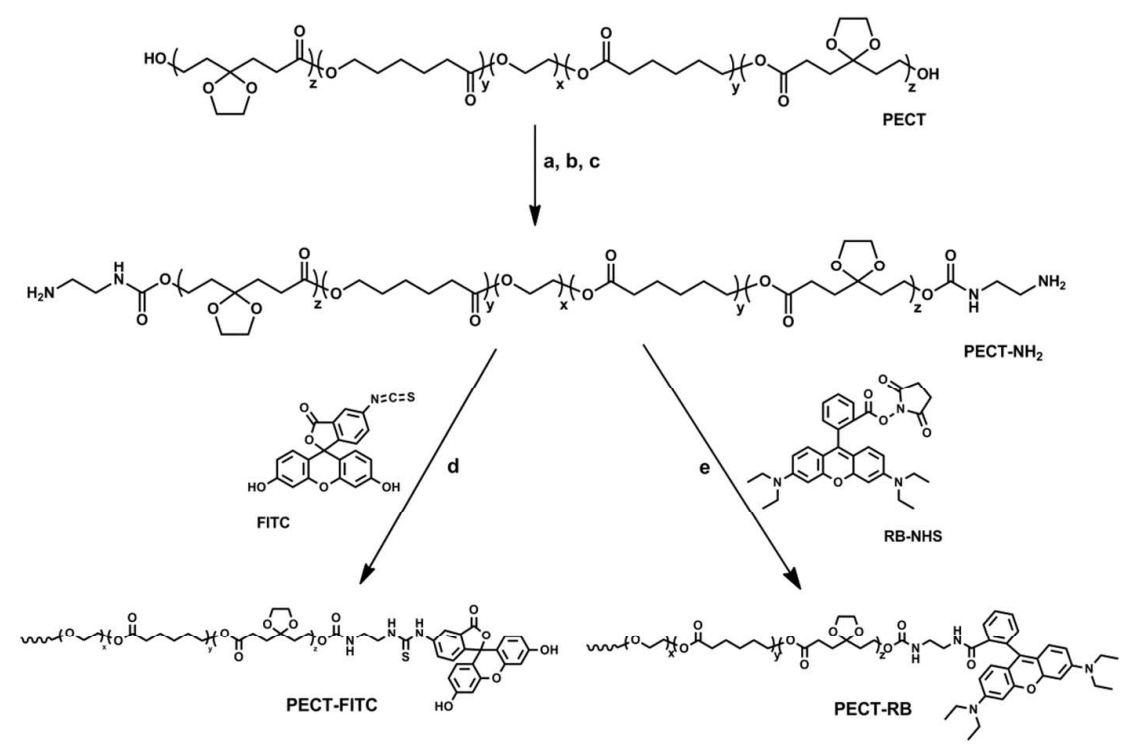

Fig. S5 The synthesis route of PECT-FITC and PECT-RB conjugates.

\section{1 Amination of PECT end hydroxyl groups}

The amination of PECT was conducted by three steps as depicted in Scheme S3 (Step a, b and c). Firstly, the terminal hydroxyl group was activated by 4-nitrophenyl chloroformate (NPC, Sigma-Aldrich) for nucleophilic substitution. Briefly, PECT (579.2 $\mathrm{mg}, 0.1 \mathrm{mmol})$ and triethylamine $(60.8 \mathrm{mg}, 0.6 \mathrm{mmol})$ were dissolved in 20 $\mathrm{mL}$ of anhydrous THF, which was cooled to $0{ }^{\circ} \mathrm{C}$ in an ice bath. Then, NPC (121 mg, $0.6 \mathrm{mmol}$ ) dissolved in $5 \mathrm{~mL}$ of anhydrous THF was added dropwise. The reaction was continued at room temperature for $24 \mathrm{~h}$. The resulted mixture was filtered and the filtrate was precipitated against ice cold anhydrous ethyl ether to obtain the PECT-NPC polymer powder, which was then dried in vacuum. Afterwards, PECT-NPC (500 mg, $0.08 \mathrm{mmol})$ and triethylamine $(81.6 \mathrm{mg}, 0.8 \mathrm{mmol})$ were 
co-dissolved in $5 \mathrm{~mL}$ anhydrous DMSO, to which N-Boc-ethylenediamine (128 mg, $0.8 \mathrm{mmol}$, Sigma-Aldrich) was subsequently added. The reaction was stirred at room temperature for $24 \mathrm{~h}$ before the solution was dialyzed against deionized water for $72 \mathrm{~h}$ with a water change frequency of every $12 \mathrm{~h}$. The PECT- $\mathrm{NH}_{2}-\mathrm{Boc}$ was obtained by lyophilization. Finally, the deprotection of Boc moiety on PECT- $\mathrm{NH}_{2}$-Boc was performed in anhydrous DCM by trifluoroacetic acid (TFA) for $2 \mathrm{~h}$ at room temperature. The reaction was quenched by addition of excess amount of sodium bicarbonate. The resulted mixture was filtered and the supernatant was precipitated against ice cold anhydrous ethyl ether to obtain the PECT-NH $\mathrm{N}_{2}$ copolymer. Ethyl ether was evaporated under vacuum.

\section{2 Synthesis and characterization of PECT-RB and PECT-FITC conjugate}

PECT copolymer was labeled with fluorescence molecules by the chemical conjugation of fluorescein isothiocyanate (FITC) or rhodamine B N-hydroxysuccinimide ester (RB-NHS) to PECT-NH $\mathrm{N}_{2}$ copolymer. Typically, PECT-NH 2 (400 mg, $0.067 \mathrm{mmol}$ ) and RB-NHS or FITC (3 eq.) were co-dissolved in $3 \mathrm{~mL}$ anhydrous DMSO in dark under the protection of argon. The reaction was conducted at room temperature for $24 \mathrm{~h}$, and the resulted mixture was dialyzed against deionized water for $72 \mathrm{~h}$ in dark with a water change frequency of every $12 \mathrm{~h}$. Finally, the fluorescence-labeled PECT copolymer, which was described as PECT-FITC or PECT-RB was obtained by lyophilization.

All polymers were characterized by ${ }^{1} \mathrm{H}$ NMR (Varian INOVA $500 \mathrm{MHz}$ and solvent DMSO- $d_{6}$ ) as shown in Fig. S5. The absorbance and emission spectra were recorded 
by Uv-vis (Purkinje General, Beijing, China) and fluorescence spectrophotometer (Varian Cary Eclipse), respectively, which were shown in Fig. S6.
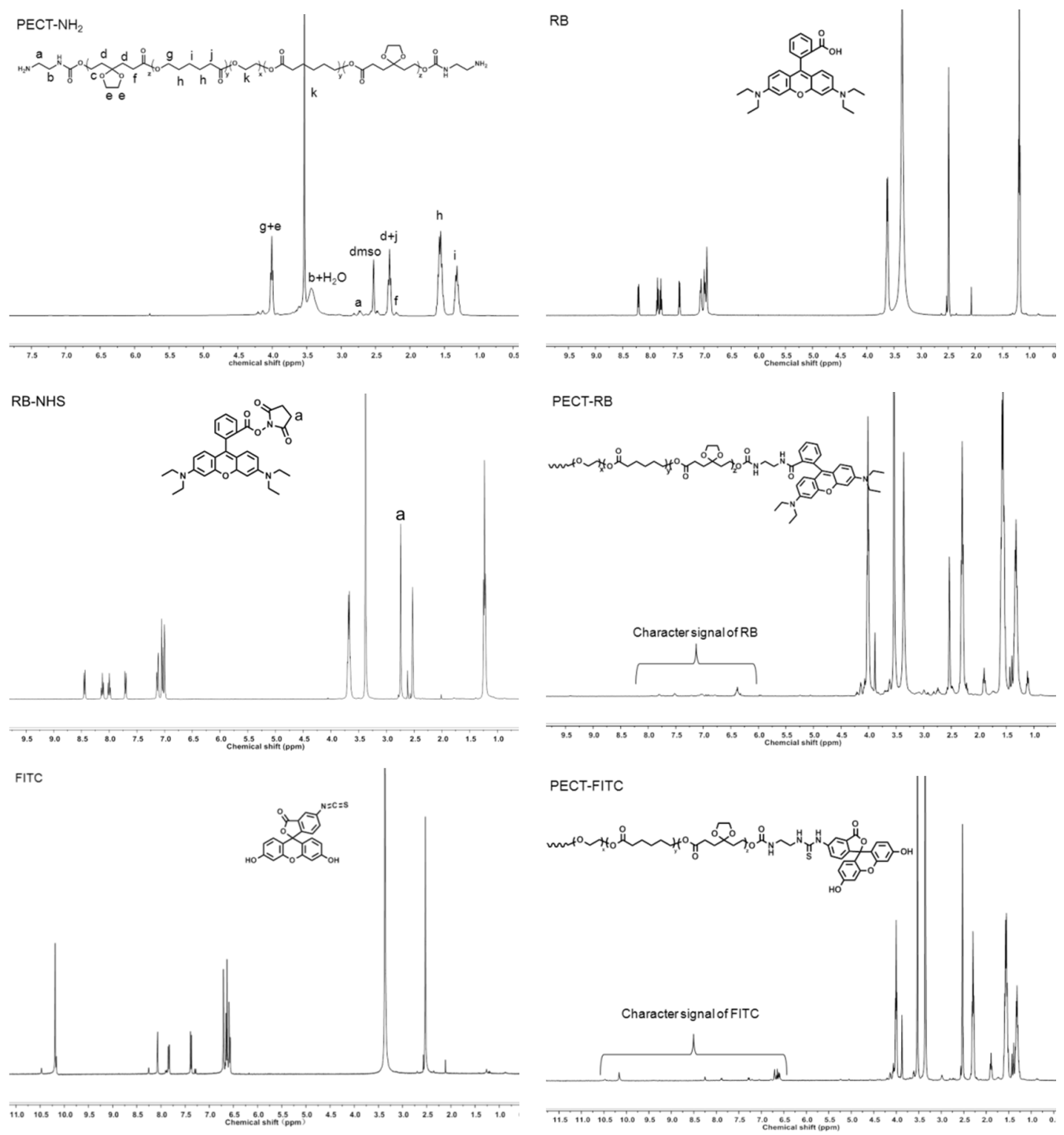

Fig. S6 ${ }^{1} \mathrm{H}$ NMR spectra of PECT-NH ${ }_{2}$, RB, RB-NHS, PECT-RB, FITC and PECT-FITC (solvent, DMSO- $\left.d_{6}\right)$. 


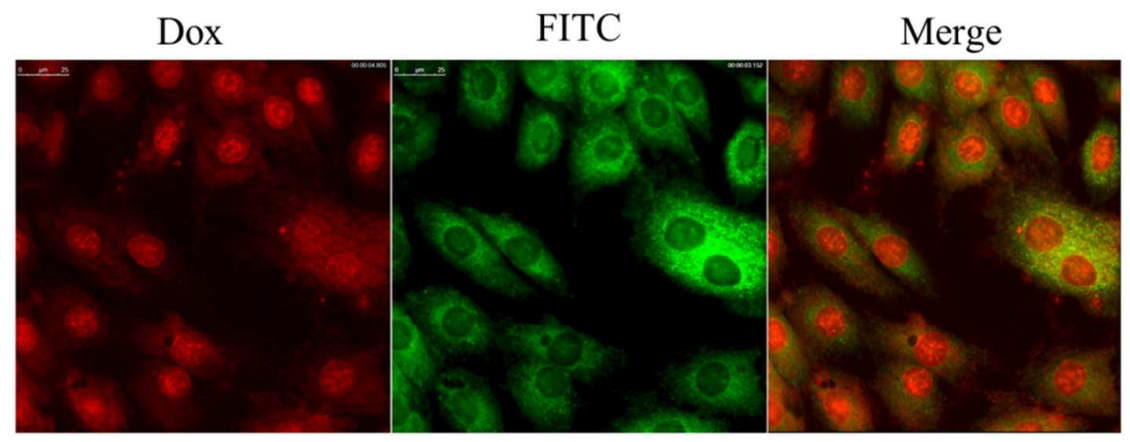

Fig. S7 The endocytosis of PECT-FITC/Dox NPs after incubation with HepG2 cells for 4 hours. (The scale bar was $25 \mu \mathrm{m}$ ).
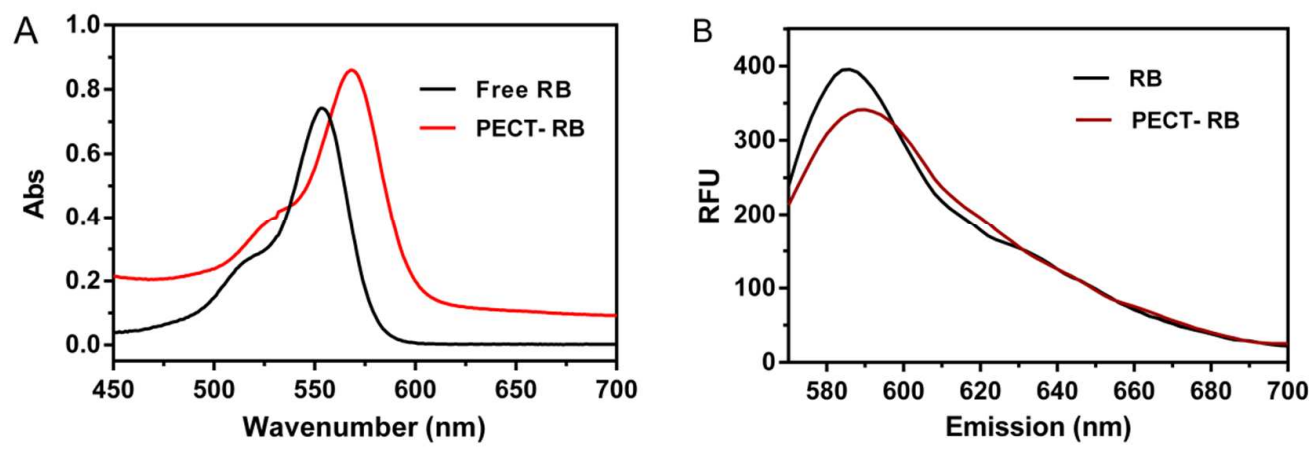

Fig. S8 (A) The absorbance profiles of free RB and PECT-RB conjugate in DMSO (dye concentration, $35 \mu \mathrm{g} / \mathrm{mL}$; PECT-RB concentration, $0.5 \mathrm{mg} / \mathrm{mL}$ ). (B) The emission spectrum of RB and PECT-RB under a excitation wavelength of $550 \mathrm{~nm}$. 